\title{
Hernia diafragmática postraumática latente, como causa de muerte súbita en un adulto mayor
}

\author{
Maikel Vargas- Sanabria, Grettchen Flores- Sandí
}

\begin{abstract}
Resumen
Se presenta el caso de un adulto mayor, conocido consumidor habitual de licor, con antecedente de politraumatismo por precipitación 11 meses atrás, e historia de sintomatología inespecífica y muerte súbita. Al realizarse la autopsia médico legal se encontró una hernia diafragmática traumática izquierda, con el estómago completamente dentro de la cavidad pleural de ese lado. La hernia diafragmática traumática es una complicación conocida y poco común del trauma toraco abdominal cerrado, que puede permanecer latente por períodos prolongados y pasar inadvertido en un adulto mayor, haciendo que su diagnóstico diferido sea un verdadero reto para el médico. Además, puede manifestarse súbitamente, en el peor de los casos, con la muerte. Desde el punto de vista médico legal, este caso constituye una excepción, pues se trata de una muerte súbita que no obedeció a causas naturales.
\end{abstract}

Descriptores: ruptura diafragmática, hernia diafragmática traumática, muerte súbita, complicaciones diferidas del trauma.

Key words: diaphragmatic injury, traumatic diaphragmatic hernia, sudden death, delayed complications of trauma.

Recibido: 19 de enero de 2007

Aceptado: 7 de mayo de 2007

\section{Caso}

Paciente masculino de 60 años de edad, con antecedente de etilismo crónico y con historia de haberse precipitado en forma accidental, en enero de 2006, en un barranco. Sufrió trauma cráneo encefálico y traumatismos múltiples en tórax, abdomen y extremidades. Fue atendido en el hospital de su área de atracción, donde se mantuvo aproximadamente 12 horas en observación, se le realizaron exámenes de rutina y fue egresado al día siguiente, con recomendaciones de que consultase en caso de presentar algún signo de alarma.

Posterior a este accidente, comenzó a presentar sintomatología inespecífica, como tos con flema y episodios ocasionales de hipo y de vómitos; más adelante, asoció dolor abdominal izquierdo. Para todo esto se le prescribió tratamiento sintomático en las ocasiones cuando consultó. A finales de diciembre de 2006, inició con un cuadro de

ISSN 0001-6002/2007/49/4/219-222

Acta Médica Costarricense, (C2007

Colegio de Médicos y Cirujanos vómitos incoercibles de contenido gástrico, asociados al final con dolor torácico izquierdo. Desistió de recibir atención médica en esta ocasión y falleció súbitamente después de desvanecerse en una silla en su vivienda.

Al examen externo del cuerpo no se encontraron alteraciones significativas, sin embargo, al abrir la cavidad torácica se evidenció la presencia de todo el estómago, rotado sobre su propio eje, a modo de vólvulo gástrico, dentro de la cavidad pleural izquierda (Figura 1), a través de un orificio de aproximadamente $10 \mathrm{~cm}$. de diámetro en el tendón central del hemidiafragma de ese lado.

\section{Discusión}

Desde el punto de vista médico legal un caso de este tipo constituye una excepción, pues se trata de una muerte súbita que no es de causas naturales, ya que el antecedente de trauma es tan lejano que hizo que el paciente aparentara un buen estado de salud por once meses. Además, la hernia diafragmática es una complicación muy poco frecuente de los traumas contusos toraco abdominales, que se diagnostica generalmente en forma aguda y que si se descompensa en forma diferida, en la mayoría de ocasiones hay tiempo de efectuar una reparación quirúrgica antes de que ocurra un desenlace fatal.

En este caso se debe considerar que la precipitación, es decir, el desplome de una víctima por debajo de su plano de sustentación, produce lesiones mínimas en piel, mientras que el daño interno es severo. ${ }^{1}$ Se producen en tórax y abdomen traumas contusos, la mayoría de las veces cerrados.

El abordaje médico inicial de un trauma contuso de tórax y abdomen está dirigido a descartar, mediante el examen físico y estudios de laboratorio y gabinete, lesiones que podrían ser letales a corto plazo, como el desgarro de la aorta torácica descendente ${ }^{2}$ y laceraciones de vísceras sólidas como el hígado y el bazo. Las lesiones traumáticas del diafragma son mucho menos frecuentes, con una incidencia de aproximadamente de un 3\% a un 5\% de los pacientes con trauma toraco abdominal severo ${ }^{3,4}$ y se presentan tanto en traumas contusos, como penetrantes o por razones iatrogénicas, ${ }^{5}$ sin embargo, su principal causa son los traumas cerrados, donde los accidentes de tránsito son los más importantes. ${ }^{6}$ Afectan principalmente a hombres jóvenes, en la tercera década de la vida, con una relación hombre-mujer de 4 a $1 .^{7}$ Las lesiones traumáticas del diafragma son raras en niños. ${ }^{8}$ 


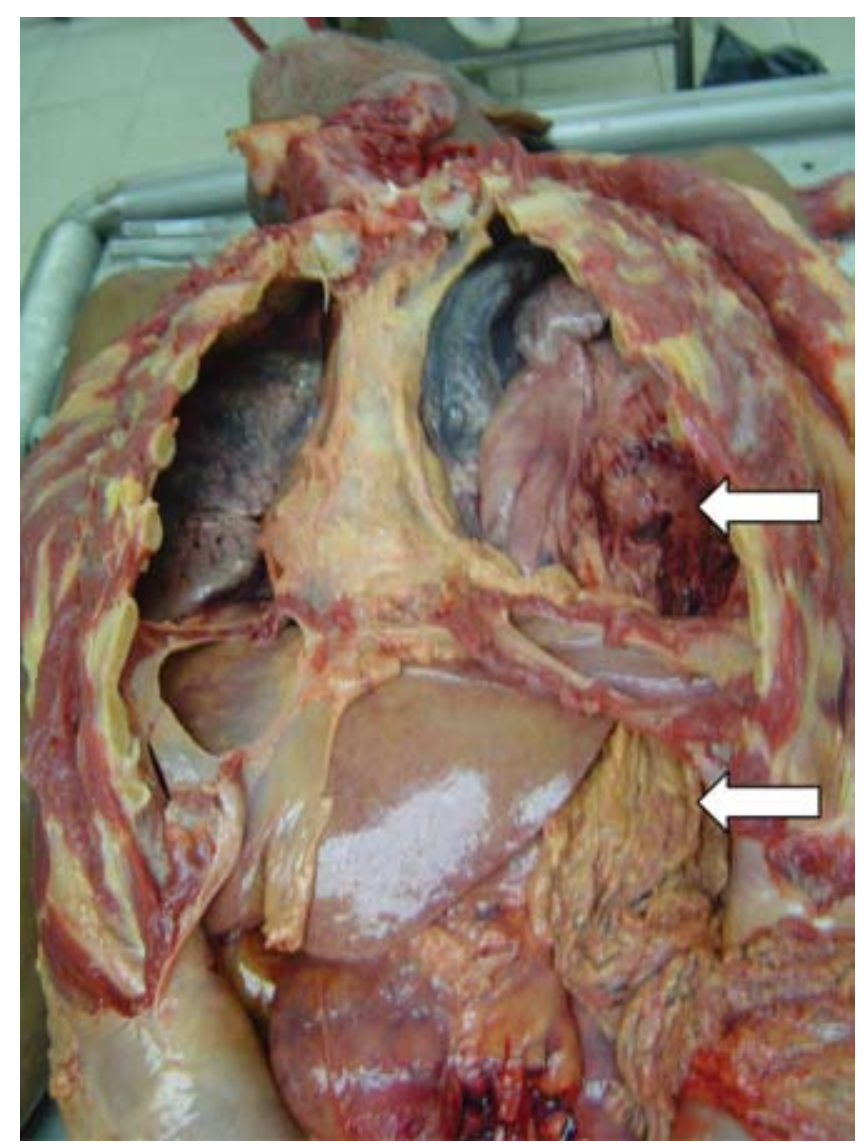

Figura 1. Se observa el viscerotórax izquierdo con el colapso pulmonar y el sitio de lesión diafragmática.

El mecanismo de lesión diafragmática en trauma contuso está dado por el impacto lateral que deforma la pared torácica y el frontal que provoca un aumento de la presión intraabdominal, pero, se requiere alta energía, lo que hace que se encuentren otras lesiones en órganos intratorácicos e intraabdominales en un 52\% a un $100 \%$ de los casos. ${ }^{9}$ En un traumatismo contuso se lesiona el diafragma en el lado izquierdo entre el $75 \%$ al $94 \%$ de los casos, tal vez porque el hígado difunde parte de la energía en el lado derecho. ${ }^{2,6} \mathrm{La}$ lesión característica es un desgarro en el tendón central, que puede ser grande ${ }^{2}$, tal y como ocurrió en el presente caso.

No obstante, el diagnóstico inicial de una lesión diafragmática es difícil, y se reportan diagnósticos tardíos en un $10 \%$ a un $61 \%$ de los casos manejados médicamente, ${ }^{7}$ pudiendo haber pacientes que se diagnostiquen hasta 50 años después de ocurrido el trauma. ${ }^{10}$ Además, comparados con pacientes jóvenes, los pacientes mayores con trauma contuso del diafragma tienen tasas significativamente más altas de radiografías de tórax normales al inicio, ${ }^{11}$ lo que hace que sean más propensos a un diagnóstico diferido.

Si la lesión cursa inadvertida en primera instancia, se pasa a una etapa latente, donde la diferencia de presiones de la cavidad abdominal con la pleural favorece la herniación de vísceras abdominales hacia el tórax. En ella, los pacientes presentan síntomas crónicos inespecíficos por meses o años ${ }^{3}$ que orientan a otras entidades como úlcera gastroduodenal, pancreatitis crónica, litiasis biliar o cardiopatía isquémica, ${ }^{12,13}$ esto hasta que se manifieste de manera inesperada, cuando los mecanismos compensatorios sean insuficientes y se produzca obstrucción o isquemia de las vísceras herniadas, lo cual tiene una mortalidad del $60 \%$ al $80 \%,{ }^{7}$ o bien, insuficiencia respiratoria por colapso pulmonar.

Por otra parte, se define como muerte súbita aquella que se manifiesta de modo brusco e inesperado en un individuo con aparente buen estado de salud. Se debe diferenciar de la muerte repentina, que es aquella que sobreviene en forma brusca en un individuo que padece una enfermedad aguda o crónica conocida. ${ }^{1}$ Se dice que es una muerte de causa natural y que es un hecho imprevisto e inesperado, cuya velocidad de instauración y desenlace son muy rápidos. ${ }^{14}$ Ocurre predominantemente en varones entre $\operatorname{los} 45$ y 75 años, con un pico máximo entre los 60 y $65 .{ }^{15}$ Se puede clasificar de acuerdo con el grupo etario, donde se considera muerte súbita del adulto a partir de los 21 años, o bien, clasificarse desde el punto de vista etiológico, según el sistema donde se presenta el evento responsable de su producción, donde el primer lugar lo ocupa el sistema cardiovascular, seguido del nervioso y el respiratorio. ${ }^{14}$ Dentro de las causas gastrointestinales se han mencionado hemorragias digestivas (úlcera gastroduodenal, várices esofágicas, tumores), infarto intestinal por trombosis mesentérica o embolia, vólvulos, estrangulación de hernias, pancreatitis y peritonitis por perforación de úlceras. ${ }^{14,16}$

En el caso descrito, el antecedente de la precipitación 11 meses atrás fue clave para establecer el mecanismo fisiopatológico (Figura 2) que condujo a la muerte, pues cumplía con los criterios o factores de causalidad médico legal para una hernia diafragmática traumática ${ }^{17}$ (Cuadro 1), sin embargo, un antecedente traumático de este tipo que no produjo fracturas ni lesiones severas que hubiesen ameritado hospitalización prolongada o intervención quirúrgica, ante un médico de un servicio de Emergencias o de primer nivel de atención puede pasar inadvertido, tal y como ocurrió. Esto es lo que hace que una hernia diafragmática postraumática latente sea una condición que haya que tener en cuenta en pacientes que consulten por sintomatología inespecífica respiratoria o gastrointestinal recurrente, y que hayan tenido historia de trauma contuso toraco-abdominal aun muchos años atrás. Cuando existe la sospecha clínica, el diagnóstico definitivo es relativamente sencillo por medio de una radiografía de tórax, lo cual permitiría una reparación quirúrgica a corto plazo.

\section{Recomendación}

Ante el antecedente de un trauma toracoabdominal cerrado significativo, aun cuando este haya ocurrido meses o años atrás, el médico debe tener la sospecha clínica de una hernia diafragmática traumática latente si el paciente presenta sintomatología gastrointestinal y respiratoria 


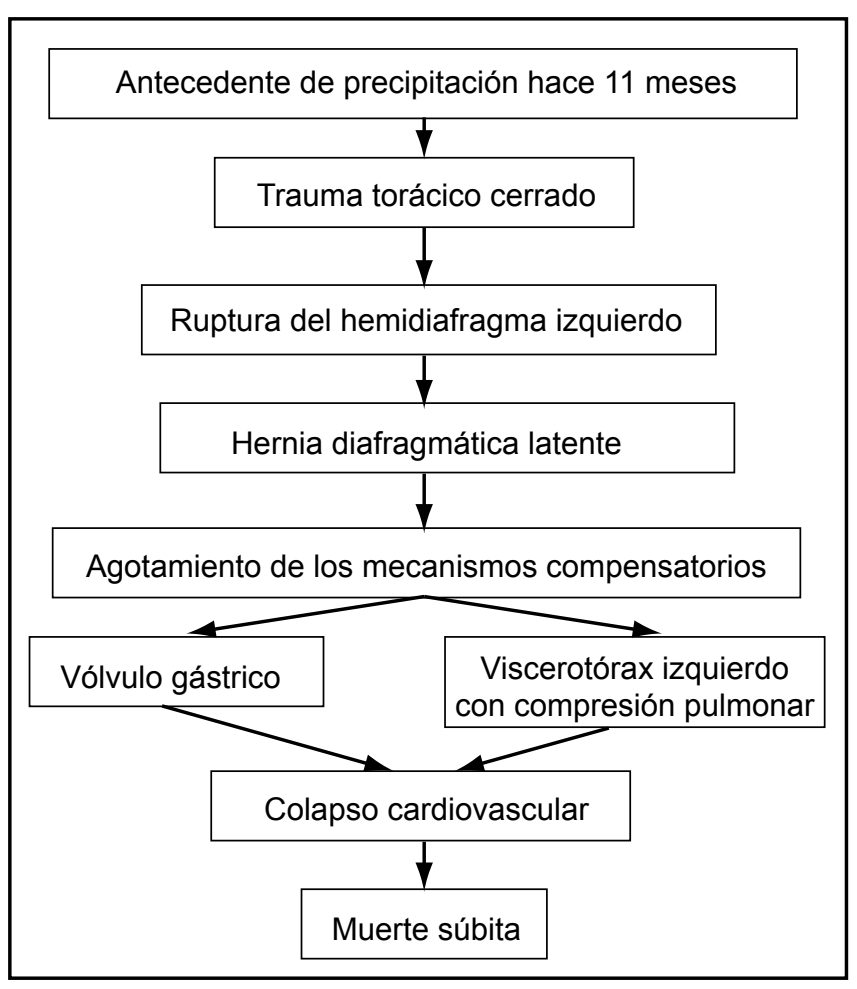

Figura 2: Esquema diagnóstico fisiopatológico de muerte súbita por hernia diafragmática postraumática latente

inespecífica a repetición, sobre todo si se trata de un adulto mayor, y debe solicitarse una radiografía de tórax para confirmar o descartar este diagnóstico.

\section{Abstract}

We present the case of a 60 year old alcoholic male who died suddenly. He had a history of having had a major fall 11 months before, suffering only external trauma. However a forensic autopsy found a trauma diaphragmatic hernia and the stomach volvulized inside the chest cavity.

Diaphragmatic hernia is an infrequent complication of thoraco-abdominal trauma, that could be latent during a long period of time, without symptoms and in an elderly patient could go unnoticed. The hernia could show itself suddenly, in the worst case even with death. A review of the literature shows the exceptionality of a traumatic event as a cause of sudden, unexpected death.

\section{Referencias}

1. Vargas E. Medicina legal. Segunda edición. México: Editorial Trillas. 2002. Pág. 97,157.

2. Brunicardi F, Andersen D, Billiar T, Dunn D, Hunter J, Pollock R. Shwartz: Principos de Cirugía. Octava edición. México: Editorial Mc Graw Hill. 2006. Pág. 158-167.

3. Ospino G, Martínez P, Valverde R. Vólvulo gástrico intratorácio secundario a hernia diafragmática izquierda crónica postraumática. Acta Méd Costarric. 2005. 47: 94-6.

\section{Cuadro 1. Factores de causalidad médico legal para una hernia diafragmática traumática}

\begin{tabular}{|c|c|}
\hline $\begin{array}{l}\text { Existencia real del } \\
\text { hecho traumático } \\
\text { denunciado }\end{array}$ & $\begin{array}{l}\text { Se sabe con certeza, que el evento } \\
\text { accidental realmente ocurrió, y no se } \\
\text { trata de una falsa denuncia, ni tampoco } \\
\text { de simulación alguna. }\end{array}$ \\
\hline Indemnidad previa & $\begin{array}{l}\text { Los antecedentes indican que al } \\
\text { momento de provocarse el trauma, el } \\
\text { paciente presentaba absoluta normalidad } \\
\text { anatómica de la zona topográfica. }\end{array}$ \\
\hline Factor etiogénico & $\begin{array}{l}\text { El factor ocasionante ha sido apropiado } \\
\text { para producir la lesión o traumatismo } \\
\text { (mecanismo medico legal de la lesión). }\end{array}$ \\
\hline $\begin{array}{l}\text { Factorcronológicoo } \\
\text { condición temporal }\end{array}$ & $\begin{array}{l}\text { Por las características del daño existente } \\
\text { se trata de una secuela que amerita tener } \\
\text { la data concordante con los hechos. }\end{array}$ \\
\hline $\begin{array}{l}\text { Factor zonal } 0 \\
\text { topográfico }\end{array}$ & $\begin{array}{l}\text { Las secuelas que presentaba asientan } \\
\text { en la misma zona anatómica que fue } \\
\text { injuriada al suceder el traumatismo. }\end{array}$ \\
\hline $\begin{array}{l}\text { Factor cuantitativo } \\
\text { o de gravedad de la } \\
\text { injuria }\end{array}$ & $\begin{array}{l}\text { El traumatismo original pudo tener } \\
\text { la fuerza o magnitud apropiada para } \\
\text { provocar una lesión diafragmática tal, } \\
\text { que pueda haber dejado la hernia que se } \\
\text { evidenció en la autopsia. }\end{array}$ \\
\hline $\begin{array}{l}\text { Sustratum objetivo, } \\
\text { cierto, no simulable }\end{array}$ & $\begin{array}{l}\text { La alteración anatómica es objetivable } \\
\text { mediante el procedimiento de autopsia. }\end{array}$ \\
\hline $\begin{array}{l}\text { Factor de } \\
\text { continuidad } \\
\text { sintomática }\end{array}$ & $\begin{array}{l}\text { Existencia de un hilo conductor que liga } \\
\text { el evento accidental traumático inicial } \\
\text { con la hernia diafragmática existente } \\
\text { considerando que desde el punto de } \\
\text { vista clínico funcional, la existencia de } \\
\text { un lapso silencioso no indica, en modo } \\
\text { alguno, que no exista relación entre uno } \\
\text { y otro de los extremos en cuestión. }\end{array}$ \\
\hline $\begin{array}{l}\text { Factor de exclusión } \\
\text { o compartido }\end{array}$ & $\begin{array}{l}\text { Ausencia de otros factores que } \\
\text { complicaron o potenciaron lo efectos del } \\
\text { traumatismo accidental descrito. }\end{array}$ \\
\hline $\begin{array}{l}\text { Factor de no } \\
\text { existencia de una } \\
\text { nueva injuria }\end{array}$ & $\begin{array}{l}\text { Ausencia de otro traumatismo } \\
\text { que asentara en la misma zona } \\
\text { anatomotopográfica que el primero, es } \\
\text { decir, que se haya sumado a este. }\end{array}$ \\
\hline
\end{tabular}

4. Jarry J, Razafindratsira T, Lepront D, Pallas G, Eggenspieler P, Dastes FD. Tension faecopneumotorax as the rare presenting feature of a traumatic diaphragmatic hernia. Ann Chir. 2006 131:48-50.

5. Eren S, Kantarci M, Okur A. Imaging of diaphragmatic rupture after trauma. Clin Radiol. 2006 61:467-77.

6. Sousa JP, Baptista JP, Martins L, Pimentel J. Traumatic diaphragmatic hernias: retrospective analysis. Rev Port Pneumol. 2006 12:225-40.

7. Llanos J, Paredes N, Schiappacasse G, Escalona A, O'Brien A. Hernia diafragmática traumática complicada: presentación como íleo. Rev Chil Rad 2005. 11:166-9.

8. Rattan KN, Magu S, Agrawal K, Ratan S. Traumatic diaphragmatic herniation. Indian J Pediatr. 2005. 72:985-6.

9. Iochum S, Ludig T, Walter F. Imaging of diaphragmatic injury: A diagnosis challenge? Radiographics. 2002. 22:103-16.

10. Singh S, Kalan M, Moreyra C, Buckman RF. Diaphragmatic rupture presenting 50 years after the traumatic event. J Trauma. 2000. 49:156-9.

11. Hsu YP, Chen RJ, Fang JF, Lin BC. Blunt diaphragmatic rupture in elderly patients. Hepatogastroenterology. 2005. 52:1752-8. 
12. Delgado J, Laurini M. Lesiones traumáticas del diafragma. Monografía del postgrado en cirugía general. Facultad de Medicina. Hospital Maciel Montevideo, Uruguay. 2001.

13. Vázquez LA, Rodríguez Y, Pila R, Pérez M. Hernia diafragmática traumática: "la gran imitadora". Archivo Médico de Camagüey 2006; 10 (5) ISSN 1025-0255. Accedido el 11 de enero de 2007 en http:// www.amc.sld.cu/amc/2006/v10n5-2006/2079.pdf

14. Patitó J, Lossetti O, Trezza F, Guzmán C, Stingo N. Tratado de medicina legal y elementos de patología forense. Primera edición. Argentina: Editorial Quórum. 2003. Pág. 296-298

15. Gisbert JA. Medicina Legal y Toxicología. Quinta edición. España: Editorial Masson. 2001. Pág. 206
16. Knight B. Medicina forense de Simpson. Segunda edición. México: Editorial El Manual Moderno. 1999. Pág. 135.

17. Kvitko LA. Factores de la causalidad médico legal. Cátedra de Medicina Legal y Deontología Médica. Facultad de Medicina. Universidad de Buenos Aires. Accesado el 17 de febrero de 2007 de http://www.mednet.org.uy/dml/bibliografia/exterior/lak-200309.htm

\title{
Tuberculosis hepática primaria como causa de fiebre de origen desconocido
}

\author{
Manuel Antonio Villalobos-Zúñiga, Júvel Quintanilla-Gallo, Charles Gourzong-Taylor.
}

\section{Resumen}

Se presenta el caso de un paciente de 29 años de edad, quien se presentó con un cuadro de fiebre, sudoración nocturna y pérdida de peso, el que inicialmente fue catalogado como dengue clásico, pero que al persistir, fue abordado como fiebre de origen desconocido. Debido a que las pruebas de laboratorio y gabinete iniciales no fueron concluyentes, fue necesario realizar una laparotomía exploratoria para obtener material histológico en el que se demostraron granulomas caseosos en el hígado y formas similares a micobacterias con las tinciones especiales. Con el tratamiento antifímico, se observó una respuesta clínica favorable, por lo que se concluye que el cuadro corresponde a una probable TB hepática.

Descriptores: tuberculosis hepática, fiebre de origen desconocido, granulomas caseosos.

Key Words: hepatic tuberculosis, fever of unknown origin, granulomas caseousus.

Recibido: 30 de mayo de 2007

Aceptado: 6 de marzo de 2007

La mayoría de los cuadros febriles agudos o subagudos corresponden a infecciones virales o bacterianas, identificables de forma sencilla con una buena historia clínica, un examen físico completo y algunos exámenes de laboratorio y gabinete rutinarios. Cuando el cuadro persiste con fiebre de $38.3^{\circ} \mathrm{C}$ o más, confirmada en varias tomas, y por al menos 3 semanas, sin diagnóstico a pesar de las indagaciones clínicas iniciales durante una semana de estudio exhaustivo intrahospitalario, la entidad clínica se denomina

ISSN 0001-6002/2007/49/4/222-225

Acta Médica Costarricense, $\odot 2007$

Colegio de Médicos y Cirujanos fiebre de origen desconocido (FOD). La nueva definición también abarca la posibilidad de estudiar al paciente de forma ambulatoria en un mínimo de 3 consultas. ${ }^{1,2}$

Para estudiar un caso de FOD como el que se expone, debe aplicarse una serie de estudios clínicos especializados, orientados a descartar enfermedades infecciosas poco comunes en frecuencia y localización, por ejemplo, tuberculosis (TB) ó micosis, enfermedades neoplásicas como el linfoma y enfermedades de estirpe reumatológico. Lo anterior se deduce de las series de casos de FOD expuestas en la bibliografía, ${ }^{3}$ donde se demuestran las causas más frecuentes de esta entidad.

\section{Caso clínico}

Paciente masculino de 29 años de edad, nicaragüense, vecino de San José, peón de construcción. Consumidor de 3 cigarrillos de tabaco diarios durante 19 años, alcohol de forma ocasional y marihuana en el pasado y en poca cantidad. Sin antecedentes patológicos conocidos. Ingreso a emergencias médicas en diciembre de 2005, por fiebre continua de 3 días de evolución, cefalea frontal, mialgias y malestar general, síntomas inespecíficos que unidos al antecedente de haber estado recientemente en Puntarenas y a la presencia de plaquetopenia en los exámenes iniciales, merecieron el diagnóstico de dengue clásico. Así fue egresado, pero la fiebre persistió por al menos 21 días, por lo cual reconsulta en enero de 2006 (4 semanas después), con los mismos síntomas, pero con un patrón de fiebre con sudoración nocturna y pérdida de peso no cuantificada, por lo que se interna bajo el diagnóstico de FOD clásica.

El examen físico reveló un paciente adelgazado, agudamente enfermo, febril $\left(40^{\circ} \mathrm{C}\right.$ en promedio), con 2 adenopatías cervicales móviles y no dolorosas, y un soplo pan-sistólico grado II, sin otros hallazgos de importancia. Se 